\title{
Continuous cerebral spinal fluid drainage associated with complications in patients admitted with subarachnoid hemorrhage
}

\author{
Clinical article
}

\author{
DaiWai M. Olson, Ph.D., R.N., ${ }^{1}$ Meg Zomorodi, Ph.D., R.N., ${ }^{2}$ \\ Gavin W. Britz, M.D., M.B.B.CH., M.P.H., ${ }^{3}$ Ali R. Zomorodi, M.D., ${ }^{4}$ \\ Anthony Amato, R.N., B.S.N., ${ }^{6}$ and Carmelo Graffagnino, M.D. ${ }^{5}$
}

${ }^{1}$ Department of Neurology and Neurotherapeutics, The University of Texas Southwestern Medical Center, Dallas, Texas; ${ }^{2}$ School of Nursing, University of North Carolina, Chapel Hill, North Carolina; ${ }^{3}$ Department of Neurosurgery, Houston Methodist Hospital, Houston, Texas; Departments of ${ }^{4}$ Neurosurgery and ${ }^{5}$ Neurology, Duke University Medical Center, Durham, North Carolina; and ${ }^{6}$ School of Nursing, Virginia Commonwealth University, Richmond, Virginia

Object. Cerebral artery vasospasm is a major cause of death and disability in patients recovering from subarachnoid hemorrhage (SAH). Although the exact cause of vasospasm is unknown, one body of research suggests that clearing blood products by CSF drainage is associated with a lower frequency and severity of vasospasm. There are multiple approaches to facilitating CSF drainage, but there is inadequate evidence to determine the best practice. The purpose of this study was to explore whether continuous or intermittent CSF drainage was superior for reducing vasospasm.

Methods. The authors performed a randomized clinical trial. Within 72 hours of admission for SAH, patients with an external ventricular drain (EVD) were randomized to undergo continuous CSF drainage with intermittent intracranial pressure (ICP) monitoring (open-EVD group) or continuous ICP monitoring with intermittent CSF drainage (monitor-ICP group).

Results. After 60 patients completed the study, an interim analysis was performed. The complication rate of $52.9 \%$ for the open-EVD group was significantly higher than the $23.1 \%$ complication rate for the monitor-ICP group (OR 3.75, 95\% CI 1.21-11.66, $\mathrm{p}=0.022$ ). These results were reported to the Data Safety and Monitoring Board and enrollment was terminated. The odds ratio of vasospasm for the open-EVD versus monitor-ICP group was not significant (OR $0.44,95 \%$ CI $0.13-1.45, \mathrm{p}=0.177)$.

Conclusions. Continuous CSF drainage with intermittent ICP monitoring is associated with a higher rate of complications than continuous ICP monitoring with intermittent CSF drainage, but there is no difference between the two types of monitoring in vasospasm. Clinical trial registration no.: NCT01169454 (clinicaltrials.gov). (http://thejns.org/doi/abs/10.3171/2013.6.JNS122403)

\section{$\begin{array}{lll}\text { KEY WoRDS } & \text { cerebral artery vasospasm } \\ \text { cerebral spinal fluid } \bullet \quad \text { neurocritical care } & \bullet & \text { subarachnoid hemorrhage } \\ \end{array}$}

$\mathrm{P}$ ATIENTS with SAH are at increased risk of delayed ischemic neurological deficit as a result of cerebral artery vasospasm, cerebral edema, changes in cerebral blood flow, and hydrocephalus, as well as secondary ischemia from hypotension, hypoxia, hypocapnia, hypercapnia, and fever., ${ }^{72,13,18}$ While the exact mechanism of

\footnotetext{
Abbreviations used in this paper: CTA = CT angiography; DSMB = Data Safety and Monitoring Board; EVD = external ventricular drain; ICP = intracranial pressure; LOS = length of stay; $\mathrm{mRS}=$ modified Rankin Scale; $\mathrm{SAH}=$ subarachnoid hemorrhage; $\mathrm{TCD}=$ transcranial Doppler
}

spasmogenesis is not completely understood, one theory ${ }^{19}$ posits that the breakdown of blood products in the CSF is a contributing factor, and several studies have linked CSF drainage with a reduction in vasospasm..$^{1,6}$

Intracranial pressure monitoring has become a cornerstone of multimodal neurological monitoring for patients with SAH. ${ }^{14}$ Unfortunately, despite the high correlation between elevated ICP and poor outcomes, research on ICP reduction is limited. ${ }^{17}$ Monitoring of ICP is a critical multidisciplinary intervention for neurologically impaired patients requiring intensive care management. It is performed using an intraparenchymal catheter, tunneled 
EVD (also called ventriculostomy catheter), or a bolt-secured intraventricular catheter. Physicians and nurses act collaboratively to deliver appropriate interventions aimed at keeping ICP within normal limits ${ }^{15}$ using a wide variety of methods for monitoring, treating, and documenting ICP values.

The most common method of facilitating CSF drainage in SAH is the use of an EVD catheter and either continuous or intermittent CSF drainage. Results from a nonrandomized observational trial of continuous versus intermittent CSF drainage have been reported in 2 separate articles describing medical and nursing-related outcomes. ${ }^{2,9}$ In the first article, Kim et al. ${ }^{9}$ reported no difference in the incidence of vasospasm between groups, but found that patients undergoing continuous drainage may have a higher rate of required shunt placement and ventricular catheter-related complications. A second study designed to compare nursing outcomes for continuous and intermittent CSF drainage found no significant differences in peak ICP, total volume or duration of CSF drainage, or the total number of EVDs used during the care of the patient. The study was unable to detect a significant difference in the rates of vasospasm between groups. ${ }^{2}$ Furthermore, Olson et al. ${ }^{16}$ reported high practice variation among 14 hospitals participating in an observational study of ICP monitoring.

These studies raised questions regarding EVD management in the clinical setting, yet their sample sizes were small and they lacked a randomized design. However, the results of the studies indicated the need for further investigation into the outcomes of continuous versus intermittent drainage. Despite the need for research in this area, there has yet to be a randomized clinical trial conducted that examines the outcomes of these 2 approaches for ICP management. Therefore, the purpose of this study was, in a randomized clinical trial, to explore how 2 different strategies for managing ICP in SAH patients impact the incidence of cerebral artery vasospasm.

\section{Methods}

The study received full approval by the Duke University Institutional Review Board as an unblinded randomized clinical trial. This study was registered with the ClinicalTrials.gov database, and its registration number is NCT01169454. Sample size estimates were based on the primary outcome of cerebral artery vasospasm using data from a published observational trial. ${ }^{11}$ A sample of 86 patients (43 per group) was determined to be sufficient to detect a significant difference. The desired number of patients was rounded upward to 100 as a more conservative estimate. An interim analysis of complications was performed after 60 patients had completed the trial. The results of the interim analysis were discussed by the DSMB, and the trial was closed to enrollment based on recommendations from the DSMB.

\section{Patients}

Adult patients were considered eligible if they were admitted to the neurocritical care unit with a diagnosis of aneurysmal SAH and if an EVD was placed by a member of the neurosurgical team within 72 hours of admission.
Patients were recruited from the neurocritical care unit after being identified by the medical team as meeting the initial eligibility requirements (that is, adults with SAH and an EVD in situ). Due to the critical illness and nature of SAH, consent was obtained from each patient's legally authorized representative.

\section{Clinical Management}

Individuals in both study groups were treated according to the institutional standard of care. Decisions regarding management of a ruptured aneurysm and treatment thresholds for CSF diversion were made by the attending neurosurgeon. Serial neurological assessments were performed every 2 hours by the nursing staff and at least daily by the physician in the neurocritical care unit. Transcranial Doppler ultrasonography was performed daily in the morning beginning on the first morning after admission. All TCD studies were reviewed and interpreted by a certified physician. The decision to undertake additional imaging (CTA or direct cerebral angiography) to assess for cerebral artery vasospasm was based on available clinical data (physical examination and TCD results). All patients received the standard of care for vasospasm prevention and treatment (for example, nimodipine, intravenous fluids, and ICU monitoring).

\section{Study Protocol}

Patients for whom consent was provided were randomized to 1 of 2 groups using a random number generator. The primary attending physician prescribed a treatment threshold (for example, treat for ICP $>15 \mathrm{~mm}$ $\mathrm{Hg}$ ), and the nursing and medical staff were provided with a printed protocol that described how and when CSF drainage should occur based on the prescribed treatment threshold (Table 1). The first group was designated as the "open-EVD" group, and staff were instructed to primarily keep the EVD open to drain CSF, but to close the EVD and monitor and record ICP at least once per hour. The second group was designated as the "monitor-ICP" group, and staff were instructed to primarily monitor ICP and only open the EVD to drain CSF if the ICP exceeded the prescribed threshold for at least 5 minutes.

\section{Measures}

Patient demographics, Hunt and Hess scores, Fisher scores, and additional baseline data were collected from all study participants. The following measures were collected as outcome measures.

Vasospasm. Vasospasm was defined as the composite of both clinically symptomatic spasm and imaging-positive spasm. Clinical vasospasm was defined as neurological deficits resulting from cerebral ischemia in the setting of imaging evidence of vasoconstriction (based on TCD, CTA, or catheter angiography). In this study, imaging positive for vasospasm was determined primarily through TCD studies. Spasm at our institution is defined as any middle cerebral artery or anterior cerebral artery velocity exceeding $130 \mathrm{~cm} / \mathrm{second}$ with a Lindegaard ratio greater than 3.0. Computed tomography angiograms and catheter angiograms were compared with those obtained at 
TABLE 1: CSF drainage protocol for each group

\begin{tabular}{cccccc}
\hline & \multicolumn{2}{c}{ Open-EVD Group } & & \multicolumn{2}{c}{ Monitor-ICP Group } \\
\cline { 2 - 3 } \cline { 5 - 5 } ICP Threshold $(\mathrm{mm} \mathrm{Hg})$ & Set EVD at $(\mathrm{mm} \mathrm{Hg})$ & Drain & & Set EVD at $(\mathrm{mm} \mathrm{Hg})$ & Drain if ICP \\
\hline 0 & 0 & continuously & -2 & $>3 \mathrm{~mm} \mathrm{Hg} \times 5 \mathrm{~min}$ \\
5 & 5 & continuously & 3 & $>8 \mathrm{~mm} \mathrm{Hg} \times 5 \mathrm{~min}$ \\
10 & 10 & continuously & 8 & $>13 \mathrm{~mm} \mathrm{Hg} \times 5 \mathrm{~min}$ \\
15 & 15 & continuously & 13 & $>18 \mathrm{~mm} \mathrm{Hg} \times 5 \mathrm{~min}$ \\
20 & 20 & continuously & 18 & $>23 \mathrm{~mm} \mathrm{Hg} \times 5 \mathrm{~min}$ \\
clamp & 25 & clamp & 25 & clamp \\
\hline
\end{tabular}

baseline, and any patient whose artery had greater than $25 \%$ narrowing compared with baseline was considered to have vasospasm. Severe spasm was defined as greater than $75 \%$ narrowing or TCD velocities greater than 200 $\mathrm{cm} / \mathrm{second}$ with a Lindegaard ratio greater than 6 .

Complications. Complications were defined as a CSF leak (any notation of CSF drainage at the insertion site), hemorrhage (intracranial hemorrhage), self device removal (any incidence of unplanned removal of the EVD other than by a physician), ventriculitis (defined as a positive CSF culture), and a nonpatent or clogged EVD (defined as any incident wherein there was no CSF drainage possible and a flat ICP waveform such that a physician was required to manipulate the EVD catheter to resume patency).

Length of Stay. Length of stay was measured by the number of days the individual was in the neurocritical care unit.

Morbidity and Mortality. Mortality was defined as a patient death occurring before hospital discharge. Morbidity was measured using mRS scores at hospital discharge. The mRS is a widely used clinical outcome measure for patients suffering from stroke. ${ }^{3,24}$ It measures the degree of disability after a stroke and has strong clinical reliability.

\section{Data Analysis}

All data were collected via chart abstraction and entered into an electronic case report form for each patient. All personal subject information was removed to protect each individual's confidentiality. All data collected were entered directly into an electronic spreadsheet and converted to SAS 9.4 for analysis. Descriptive statistics were performed to describe measures of central tendency. Nonparametric data were explored using the WilcoxonMann-Whitney test. Hypothesis testing was conducted with a level of significance set at alpha $=0.05$.

\section{Results}

The study enrolled 60 patients with SAH; 34 were randomized to the open-EVD group and 26 to the monitor-ICP group. There were 15 individuals who met inclusion criteria but declined participation (consent failure). There were no significant intergroup differences in base- line characteristics, severity of illness, or aneurysm treatment (Table 2). In the open-EVD group, patients' mean age was 57.2 years and $73.5 \%$ were female; these characteristics were similar to those in the monitor-ICP group in which mean age was 59.4 years and $69.3 \%$ were female ( $p=0.501$ and 0.720 , respectively). Subjects in both groups were predominantly non-Hispanic (over 95\%) and Caucasian (over 60\%). Injury severity was measured upon admission; the median Hunt and Hess scores were 3 and $3(p=0.1096)$ and the median Fisher scores were 3 and $3(\mathrm{p}=0.6177)$, respectively, for the open-EVD group and the monitor-ICP group. In the open-EVD group, 15 patients underwent open surgical clipping of the aneurysm and 15 underwent endovascular coiling. In the monitorICP group, 10 patients underwent open surgical clipping of the aneurysm and 10 underwent endovascular coiling.

The primary outcome of the study was composite vasospasm, namely, the presence of both clinically symptomatic spasm and imaging-positive spasm (Table 3). Vasospasm monitoring by TCD ultrasonography was performed daily in all patients, and vasospasm was defined as a Lindegaard ratio greater than 3.0, with a mean TCD velocity greater than $130 \mathrm{~cm} / \mathrm{second}$ (mild), greater than $150 \mathrm{~cm} / \mathrm{second}$ (moderate), and greater than $200 \mathrm{~cm} / \mathrm{sec}-$ ond (severe). Using TCD criteria, there was no significant

TABLE 2: Baseline demographics

\begin{tabular}{lccc}
\hline \multicolumn{1}{c}{ Variable } & $\begin{array}{c}\text { Open-EVD } \\
\mathrm{n}=34\end{array}$ & $\begin{array}{c}\text { Monitor-ICP } \\
\mathrm{n}=26\end{array}$ & $\mathrm{p}$ Value \\
\hline mean age (SD) & $57.17(13.33)$ & $59.38(11.32)$ & 0.5005 \\
sex & & & 0.7198 \\
$\quad$ male & $26.5 \%$ & $30.7 \%$ & \\
$\quad$ female & $73.5 \%$ & $69.3 \%$ & \\
ethnicity & & & 0.8497 \\
$\quad$ Hispanic & $2.9 \%$ & $3.8 \%$ & \\
$\quad$ non-Hispanic & $97.1 \%$ & $96.2 \%$ & \\
race & & & 0.9156 \\
$\quad$ Caucasian & $64.8 \%$ & $61.5 \%$ & \\
African American & $29.4 \%$ & $34.6 \%$ & \\
Asian & $2.9 \%$ & 0 & \\
$\quad$ Native American & $2.9 \%$ & $3.9 \%$ & \\
severity (median) & & & \\
$\quad$ Hunt \& Hess score & 3 & 3 & 0.1096 \\
Fisher score & 3 & 3 & 0.6177 \\
\hline
\end{tabular}




\section{Continuous versus intermittent CSF drainage}

TABLE 3: Frequency and percentages for dichotomous outcomes

\begin{tabular}{lccccc}
\hline \multicolumn{1}{c}{ Variable } & $\begin{array}{c}\text { Open-EVD } \\
\mathrm{n}=34\end{array}$ & $\begin{array}{c}\text { Monitor-ICP } \\
\mathrm{n}=26\end{array}$ & Odds Ratio & $95 \% \mathrm{Cl}$ & $\mathrm{p}$ Value \\
\hline vasospasm & & & & & \\
any method & $22(64.7 \%)$ & $21(80.8 \%)$ & 0.44 & $0.13-1.45$ & 0.1768 \\
by TCD & $21(61.7 \%)$ & $18(69.2 \%)$ & 0.72 & $0.24-2.12$ & 0.5486 \\
by angiogram & $7(20.6 \%)$ & $9(34.6 \%)$ & 0.49 & $0.15-1.56$ & 0.2274 \\
by CTA & $13(38.2 \%)$ & $10(38.5 \%)$ & 0.99 & $0.347-2.831$ & 0.9857 \\
complication* & & & & & \\
any complication & $18(52.9 \%)$ & $6(23.1 \%)$ & 3.75 & $1.21-11.66$ & 0.0223 \\
nonpatent EVD & $15(44.1 \%)$ & $3(11.5 \%)$ & 4.35 & $1.18-16.10$ & 0.0276 \\
ventriculitis & $6(17.6 \%)$ & $1(3.8 \%)$ & 5.36 & $0.60-47.57$ & 0.1322 \\
self device removal & $3(8.8 \%)$ & $2(7.7 \%)$ & 1.16 & $0.18-7.51$ & 0.8753 \\
CSF leak/hemorrhage & $5(14.7 \%)$ & $1(3.8 \%)$ & 4.31 & $0.47-39.39$ & 0.1956 \\
shunt placement & $3(8.8 \%)$ & $5(19.2 \%)$ & 0.41 & 0.09 & 0.2503 \\
discharge mRS $<2$ & $7(20.6 \%)$ & $5(19.2 \%)$ & 1.09 & $0.30-3.92$ & 0.8968 \\
discharge mRS $<3$ & $11(32.3 \%)$ & $9(34.6 \%)$ & 0.90 & $0.30-2.66$ & 0.8539 \\
discharge mRS $>4$ & $15(44.1 \%)$ & $8(30.8 \%)$ & 1.78 & $0.61-5.20$ & 0.2941 \\
died & $8(23.5 \%)$ & $3(11.5 \%)$ & 2.36 & $0.56-9.96$ & 0.2430 \\
\hline
\end{tabular}

* Categories are not mutually exclusive.

difference in the odds of experiencing composite vasospasm for the open-EVD group versus the monitor-ICP group $(61.7 \%$ vs $69.2 \%$, respectively; OR $0.72,95 \% \mathrm{CI}$ 0.24-2.12). Computed tomography angiography was performed at the discretion of the neurosurgeon, based on clinical examination results and TCD values. There was no significant difference in the odds of experiencing vasospasm as evidenced by CTA for the open-EVD group versus the monitor-ICP group $(38.2 \%$ vs $38.5 \%$, respectively; OR 0.99, 95\% CI 0.35-2.83). Direct catheter-based cerebral artery angiography was performed at the discretion of the neurosurgeon, based on clinical examination results and/or TCD/CTA values. With evidence of vasospasm provided by angiography, there was no significant difference in the odds of experiencing vasospasm for the open-EVD group versus the monitor-ICP group $(20.6 \%$ vs $34.6 \%$, respectively; OR 0.49 , 95\% CI $0.15-1.56$ ). Grouping all diagnostic methods, there was no significant difference in the odds of experiencing vasospasm between the open-EVD group and the monitor-ICP group (64.7\% and $80.8 \%$, respectively; OR $0.44,95 \%$ CI $0.13-1.45$ ).

Complications identified as being associated with EVD placement included CSF leakage, hemorrhage, self device removal, ventriculitis, and a nonpatent EVD (Table 3). Patients in the open-EVD group had higher odds of experiencing any complication $(52.9 \%$ vs $23.1 \%$, respectively; OR $3.75,95 \%$ CI 1.21-11.66) than those in the monitor-ICP group. A nonpatent EVD was defined as one that lost patency such that a neurosurgeon had to flush or replace the system. Patients in the open-EVD group had higher odds of having a nonpatent EVD than those in the monitor-ICP group (44.1\% vs $11.5 \%$, respectively; OR 4.35, 95\% CI 1.18-16.10). Ventriculitis was defined as a positive CSF culture. Ventriculitis was confirmed in $6(17.6 \%)$ of 34 patients in the open-EVD group and 1
(3.8\%) of 26 patients in the monitor-ICP group, respectively (OR 5.36, 95\% CI $0.60-47.57$ ). Of the 18 patients with nonpatent EVDs, 5 had a positive CSF culture (OR 7.96, 95\% CI 1.33-44.50). The composite of ventriculitis and/or nonpatent EVD was noted in $17(50 \%)$ of 34 patients in the open-EVD group and $4(15.4 \%)$ of 26 patients in the monitor-ICP group, respectively (OR 5.50, 95\% CI $1.56-19.38)$.

There were no intergroup differences for secondary outcomes associated with CSF drainage (Table 4). In the open-EVD group, the mean values for highest ICP $(41.65 \mathrm{~mm} \mathrm{Hg})$, daily CSF drainage $(124.16 \mathrm{ml})$, number of EVDs placed (1.26), and number of days the EVD remained in situ (12.79) were not statistically different from those of the monitor-ICP group (39.96 mm Hg, 105.02 ml, 1.19 EVDs, and 12.69 days, respectively). The mean LOS in the open-EVD group was 15.93 days and was not significantly different from the mean LOS in the monitor-ICP group (16.06 days; $p=0.948)$. Mean $m R S$ scores measured at hospital discharge in the open-EVD group (3.65) were not significantly different from those in the monitor-ICP group ( $3.27 ; \mathrm{p}=0.466)$.

\section{Discussion}

The results of this randomized clinical trial extend the findings from the observational trial of continuous and intermittent CSF drainage for patients with SAH. ${ }^{2,9}$ The significantly higher complication rate associated with continuous CSF drainage in the open-EVD group resulted in a decision by the DSMB to close the study to further enrollment. The most serious complication was a positive CSF culture. The percentage of patients with ventriculitis was clinically relevant but not statistically significantly different $(17.6 \%$ in the open-EVD group 
D. M. Olson et al.

TABLE 4: Mean values and standard deviations for secondary outcomes

\begin{tabular}{lccc}
\hline \multicolumn{1}{c}{ Variable } & $\begin{array}{c}\text { Open-EVD } \\
\mathrm{n}=34\end{array}$ & $\begin{array}{c}\text { Monitor-ICP } \\
\mathrm{n}=26\end{array}$ & $\mathrm{p}$ Value \\
\hline highest recorded ICP $(\mathrm{ml})$ & $41.65(18.2)$ & $39.96(13.67)$ & 0.6950 \\
total CSF output $(\mathrm{ml})$ & $1734.50(1650.47)$ & $1448.62(1086.36)$ & 0.4475 \\
daily CSF output (ml) & $124.16(81.37)$ & $105.02(52.07)$ & 0.3001 \\
EVD dwell time (days) & $12.79(8.92)$ & $12.69(5.74)$ & 0.9598 \\
number of EVDs & $1.26(0.80)$ & $1.19(0.40)$ & 0.6715 \\
LOS in ICU (days) & $15.93(0.37)$ & $16.06(6.45)$ & 0.9477 \\
discharge mean mRS & $3.65(2.09)$ & $3.27(1.82)$ & 0.4664 \\
\hline
\end{tabular}

and $3.8 \%$ in the monitor-ICP group, $\mathrm{p}=0.132$ ). The most common complication was the EVD losing patency and requiring manipulation (flushing or replacing the EVD) by a neurosurgeon to maintain patency. Important in the DSMB decision to stop enrollment was the strong association (alpha $=0.01$ ) between infection and a nonpatent EVD, without regard to group assignment (OR 7.96), combined with the statistically significantly higher odds of a nonpatent EVD in the open-EVD group (OR 4.35).

The infection rate in the open-EVD group is nearly twice the reported average. Kim et al. summarized the results from 17 studies and reported an average infection rate of $9.5 \%$ (range $0 \%-22 \%$ )..$^{10}$ In the observational study of continuous and intermittent CSF drainage, $12.5 \%$ of patients who underwent continuous CSF drainage versus $0 \%$ of those who underwent intermittent drainage were diagnosed with ventriculitis. ${ }^{9}$ To fully explore the relative risk of infection for patients undergoing continuous CSF drainage, we combined the sample populations from the randomized and observational studies conducted at our institution. The odds of ventriculitis developing in the open-EVD group (15.5\%, or 9 of 58 patients) compared with the monitorICP group rate $(2.6 \%$, or 1 of 39 patients) remained clinically relevant but not statistically significant with a wide confidence interval (OR 6.98, 95\% CI 0.947-57.513). The overall rates of ventriculitis of $11.7 \%$ (7 of 60 patients) in the randomized study and $8.1 \%$ ( 3 of 37 patients) in the observational study at our institution are similar to reported averages..$^{10}$ This finding further supports the belief that the difference in infection rates is related to group differences and not institutional differences.

The association between the higher number of nonpatent EVDs and ventriculitis in the open-EVD group may be explained by a higher likelihood of contamination each time the neurosurgeon accesses the closed sterile system during flushing to restore flow. Maintaining a patent lumen is vital to proper functioning of EVDs. In both the current randomized study and the previous observational study, the rate of nonpatent EVDs (those that required flushing to resume patency) was higher in the open-EVD group. ${ }^{9}$ Excluding complications that are potentially patient dependent (self device removal and CSF leakage or hemorrhage) did not reduce the higher odds of complication (OR 5.50, as reported above) in the openEVD group.

A power analysis performed prior to the present study determined that a sample size of 86 subjects would be sufficient to explore the impact of the 2 different drainage mechanisms on vasospasm rates. The diagnosis of vasospasm was made by the neurointensivist based on the combination of clinical examination and TCD results. Serial neurological examinations and TCD studies were completed at least daily for all patients. Not all patients in the study underwent CTA and/or direct cerebral angiography to diagnose vasospasm, but, consistent with the literature, there were no cases where vasospasm was identified by CTA or angiography, and was also not identified by the combination of neurological examination and TCD ultrasonography. ${ }^{8}$

Although the trial was stopped early and there was no statistically significant difference in the primary outcome of vasospasm, there was a trend toward significance in favor of the open-EVD group. It is not possible to know what the final rates of vasospasm would have been if the trial had been allowed to continue to full enrollment. However, one could speculate that the trend toward significance suggests that, if a mechanism could be determined to reduce the risk of complications from open-EVD, there is a potential for reducing the risk of vasospasm and thereby benefiting the patient. The finding of clinically significant higher rates of vasospasm is, however, dependent on the assumption that vasospasm can be equally well identified by angiography or TCD ultrasonography (Table 3). This assumption is generally considered not valid; TCD ultrasonography is a poor surrogate for diagnosing vasospasm. ${ }^{5}$

The lack of observed difference in secondary outcomes such as LOS in the ICU and functional status at discharge may be the result of stopping the trial early. However, the lack of intergroup differences is consistent with results from the observational study. ${ }^{9}$ Furthermore, the standard of care at our institution is a minimum LOS of 14 days for all SAH patients (survivors), thus creating a potential bias toward longer LOS. To fully explore functional outcomes, mRS scores were dichotomized first based on dependence (mRS score of 1 vs 2-6) and then based on extremely poor outcome (mRS score of 5-6) under the assumption that patients view a major stroke as an outcome as bad as or worse than death. ${ }^{20}$

\section{Limitations}

There are several important limitations that should be recognized. First, the sample size of 60 patients is not sufficient to reject the null hypothesis of no difference in 
vasospasm by treatment group. The study found a clinically, but not statistically significant, intergroup difference in ventriculitis. The study was not powered for this outcome, and future research may benefit from randomized or quasi-experimental designs powered to detect a difference in ventriculitis. There was no effort to blind the practitioners (nurses or doctors caring for the patient) to the EVD being open or closed to CSF drainage, and this may have introduced bias that is unmeasured.

We do not have images of the brain obtained with CT or MRI (diffusion-weighted imaging) to look for evidence of delayed ischemic injury; such images might be more sensitive for detecting the effects of vasospasm than clinical examination alone. ${ }^{22,23}$ Carrera et al. ${ }^{4}$ have shown that strokes occur even in areas where there was no TCD ultrasonography-demonstrated spasm. If CSF drainage helps with vasospasm or ischemic injury, we might have been able to see it with an MR image.

The inclusion criteria for this study were defined only as aneurysmal SAH requiring EVD placement based on the discretion of the attending neurosurgeon. This broad inclusion criterion increases the external validity of the study, but the limited internal validity is a potential bias. Future research may find increased benefit or harm associated with different inclusion criteria (for example, only including patients with high Fisher scores). The use of $\mathrm{mRS}$ scores at discharge may not accurately reflect functional outcomes in this population given that recovery from CNS injury may be delayed. It is generally accepted that early assessment of poststroke disability is less informative than are 3- or 6-month outcome data. ${ }^{21}$ Having 90-day outcome data would be beneficial to understanding the full impact of the intervention.

It is a recognized limitation that groups were unequal in size. Only 26 patients were randomized to the monitorICP group compared with 34 in the open-EVD group. To remain conservative and control for lost-to-follow-up rates, randomization was performed using a random number table and randomization without replacement with an estimated sample size of 100 subjects. The study was terminated early after the DSMB recommended that the study should stop enrollment because the results of the interim data analysis were clinically significant in finding a higher risk of complication among patients randomized to the open-EVD group.

\section{Conclusions}

Continuous CSF drainage with only intermittent ICP monitoring is associated with a significantly greater risk of complications than CSF drainage triggered by ICP treatment threshold. The clinically relevant, but not statistically significant, difference in vasospasm deserves greater attention. Future research should focus on determining the optimal treatment threshold to maximize CSF drainage for patients with SAH who receive ICP monitoring via EVD.

\section{Disclosure}

Research funding was received from the Medtronic Corporation and the Duke Translational Research Institute.

Author contributions to the study and manuscript preparation include the following. Conception and design: Olson, M Zomorodi, AR Zomorodi, Britz, Graffagnino. Acquisition of data: Olson, Amato. Analysis and interpretation of data: Olson, Britz, Graffagnino. Drafting the article: Olson, Britz. Critically revising the article: all authors. Reviewed submitted version of manuscript: all authors. Approved the final version of the manuscript on behalf of all authors: Olson. Statistical analysis: Olson. Administrative/technical/material support: Olson. Study supervision: Olson, Graffagnino.

\section{Acknowledgments}

The authors would like to acknowledge Ms. Kristina Riemen and the DSMB: Drs. Brad Kolls (chair), Daniel Laskowitz, and Keith Dombrowski.

\section{References}

1. Al-Tamimi YZ, Bhargava D, Feltbower RG, Hall G, Goddard AJ, Quinn AC, et al: Lumbar drainage of cerebrospinal fluid after aneurysmal subarachnoid hemorrhage: a prospective, randomized, controlled trial (LUMAS). Stroke 43:677-682, 2012

2. Amato A, Britz GW, James ML, Graffagnino C, Zomorodi AR, Zomorodi ME, et al: An observational pilot study of CSF diversion in subarachnoid haemorrhage. Nurs Crit Care 16:252-260, 2011

3. Banks JL, Marotta CA: Outcomes validity and reliability of the modified Rankin scale: implications for stroke clinical trials: a literature review and synthesis. Stroke 38:1091-1096, 2007

4. Carrera E, Schmidt JM, Oddo M, Fernandez L, Claassen J, Seder D, et al: Transcranial Doppler for predicting delayed cerebral ischemia after subarachnoid hemorrhage. Neurosurgery 65:316-324, 2009

5. Frontera JA, Fernandez A, Schmidt JM, Claassen J, Wartenberg KE, Badjatia N, et al: Defining vasospasm after subarachnoid hemorrhage: what is the most clinically relevant definition? Stroke 40:1963-1968, 2009

6. Fugate JE, Rabinstein AA, Wijdicks EF, Lanzino G: Aggressive CSF diversion reverses delayed cerebral ischemia in aneurysmal subarachnoid hemorrhage: a case report. Neurocrit Care 17:112-116, 2012

7. Ionita CC, Baker J, Graffagnino C, Alexander MJ, Friedman $\mathrm{AH}$, Zaidat OO: Timing of symptomatic vasospasm in aneurysmal subarachnoid hemorrhage: the effect of treatment modality and clinical implications. J Stroke Cerebrovasc Dis 19:110-115, 2010

8. Ionita CC, Graffagnino C, Alexander MJ, Zaidat OO: The value of CT angiography and transcranial doppler sonography in triaging suspected cerebral vasospasm in SAH prior to endovascular therapy. Neurocrit Care 9:8-12, 2008

9. Kim GS, Amato A, James ML, Britz GW, Zomorodi A, Graffagnino C, et al: Continuous and intermittent CSF diversion after subarachnoid hemorrhage: a pilot study. Neurocrit Care 14:68-72, 2011

10. Kim JH, Desai NS, Ricci J, Stieg PE, Rosengart AJ, Härtl R, et al: Factors contributing to ventriculostomy infection. World Neurosurg 77:135-140, 2012

11. Lipsey MW: Design Sensitivity: Statistical Power for Experimental Research. Newbury Park, CA: Sage Publications, 1990

12. Macdonald RL: Cerebral Vasospasm: Advances in Research And Treatment. New York: Thieme, 2005

13. Macdonald RL, Hunsche E, Schüler R, Wlodarczyk J, Mayer SA: Quality of life and healthcare resource use associated with angiographic vasospasm after aneurysmal subarachnoid hemorrhage. Stroke 43:1082-1088, 2012

14. Olson DM: Multimodal neurological monitoring, in Kaplow R, Hardin SR (eds): Critical Care Nursing: Synergy for Optimal Outcomes. Boston: Jones and Bartlett, 2007, pp 359-374 
15. Olson DM, Bader MK, Dennis C, Mahanes D, Riemen K: Multicenter pilot study: safety of automated chest percussion in patients at risk for intracranial hypertension. J Neurosci Nurs 42:119-127, 2010

16. Olson DM, Lewis LL, Bader MK, Bautista C, Malloy R, Riemen KE, et al: Practice variation associated with intracranial pressure monitoring. J Neurosci Nurs 45:186-193, 2013

17. Olson DM, McNett MM, Livesay S, Le Roux PD, Suarez JI, Bautista C: Neurocritical care nursing research priorities. Neurocrit Care 16:55-62, 2012

18. Pluta RM, Hansen-Schwartz J, Dreier J, Vajkoczy P, Macdonald RL, Nishizawa S, et al: Cerebral vasospasm following subarachnoid hemorrhage: time for a new world of thought. Neurol Res 31:151-158, 2009

19. Provencio JJ, Fu X, Siu A, Rasmussen PA, Hazen SL, Ransohoff RM: CSF neutrophils are implicated in the development of vasospasm in subarachnoid hemorrhage. Neurocrit Care 12:244-251, 2010

20. Samsa GP, Matchar DB, Goldstein L, Bonito A, Duncan PW, Lipscomb J, et al: Utilities for major stroke: results from a survey of preferences among persons at increased risk for stroke. Am Heart J 136:703-713, 1998

21. Saver JL, Filip B, Hamilton S, Yanes A, Craig S, Cho M, et al: Improving the reliability of stroke disability grading in clinical trials and clinical practice: the Rankin Focused Assessment (RFA). Stroke 41:992-995, 2010
22. Vergouwen MD, Ilodigwe D, Macdonald RL: Cerebral infarction after subarachnoid hemorrhage contributes to poor outcome by vasospasm-dependent and -independent effects. Stroke 42:924-929, 2011

23. Vergouwen MD, Vermeulen M, van Gijn J, Rinkel GJ, Wijdicks EF, Muizelaar JP, et al: Definition of delayed cerebral ischemia after aneurysmal subarachnoid hemorrhage as an outcome event in clinical trials and observational studies: proposal of a multidisciplinary research group. Stroke 41:23912395,2010

24. Wilson JT, Hareendran A, Hendry A, Potter J, Bone I, Muir KW: Reliability of the modified Rankin Scale across multiple raters: benefits of a structured interview. Stroke 36:777-781, 2005

Manuscript submitted January 9, 2013

Accepted June 24, 2013.

Please include this information when citing this paper: published online August 20, 2013; DOI: 10.3171/2013.6.JNS122403.

Address correspondence to: DaiWai M. Olson, Ph.D., R.N., Department of Neurology and Neurotherapeutics, University of Texas Southwestern Medical Center, 5323 Harry Hines Blvd., Dallas, TX 75390-8897. email: DaiWai.Olson@UTSouthwestern.edu. 